\title{
Self-induced frequency scanning and distributed Bragg reflection in semiconductor lasers with phase-conjugate feedback
}

\author{
Mark Cronin-Golomb \\ Ortel Corporation, 2015 West Chestnut Street, Alhambra, California 91803 \\ Amnon Yariv \\ California Institute of Technology, Pasadena, California 91125
}

Received December 16, 1985; accepted April 9, 1986

\begin{abstract}
A GaAlAs semiconductor laser with feedback from a barium titanate photorefractive ring passive phase-conjugate mirror can be made to perform repeating or nonrepeating frequency scans over a 10-nm range toward either the blue or the red. The direction of scanning and whether the scans repeat may be controlled by adjusting the overlap of the interaction beams in the crystal. This overlap region may be adjusted so that the diode frequency spectrum, originally occupying about 10 longitudinal modes, scans and narrows as the conjugate signal builds up, coming to rest often in one, but sometimes two or three, longitudinal modes as a result of self-generated distributed-feedback effects. We also report similar effects caused by feedback from the total-internal-reflection passive phaseconjugate mirror. The alignment-control mechanism of the ring mirror is, however, not available in this case.
\end{abstract}

The observation of frequency self-scanning and linenarrowing effects in dye lasers ${ }^{1-4}$ under feedback from photorefractive passive phase-conjugate mirrors ${ }^{5-7}$ (PPCM's) shows considerable promise for spectroscopic applications. ${ }^{8}$ While the published experimental results were obtained using the total-internal-reflection $\mathrm{PPCM}^{6}$ (cat mirror), detuning has also been observed in dye lasers with feedback from the ring PPCM. A ring PPCM using $\mathrm{BaTiO}_{3}$ was recently operated with light from GaAlAs lasers. ${ }^{9}$ This Letter reports observations of similar frequency-scanning effects in GaAlAs semiconductor lasers with feedback from the ring PPCM. The direction of the scan, and whether it occurs repetitively, may be controlled by adjusting the ring PPCM alignment. Self-induced distributed-feedback effects can cause narrowing of the spectrum to a single longitudinal mode. We have also seen frequency-scanning effects in these lasers caused by feedback from a cat mirror.

Figure 1 is a schematic of the experiment consisting of a Michelson interferometer with one regular mirror and one $\mathrm{BaTiO}_{3}$ ring $\mathrm{PPCM}$. The reference arm of the interferometer is deliberately slightly misaligned so that light from this arm is not fed back into the laser and does not spuriously affect the laser spectrum. The absence of these unwanted feedback effects was verified by noting that blocking the ordinary mirror did not influence the laser spectrum. The misalignment also facilitates the observation of clear interference fringes. The diode laser used is a buried-heterostructure GaAlAs window laser ${ }^{10}$ with high-reflectivity rear facet and antireflection-coated front facet delivering $29 \mathrm{~mW}$ of power at $80-\mathrm{mA}$ injection current (20 $\mathrm{mW}$ after the collimating objective). The thresh- old current $I_{\text {th }}$ of the laser is approximately $15 \mathrm{~mA}$. The spot size at the crystal is about $1 \mathrm{~mm}$. The output of the interferometer is directed to a grating monochromator with a video display. The function of the monochromator is twofold. First, it is used to monitor the laser spectrum. Second, it allows the interferometer fringes to be spectrally resolved, so that even though the laser may be lasing in many longitudinal modes and no special care is taken to match the relative delay of the two interferometer arms the fringes are still easily seen and have the high visibility associated with the long coherence length of the individual modes. These interferometric fringes are used to monitor any spontaneous motion of the photorefractive index gratings. One of the feedback mirrors of the ring PPCM is mounted on an electrically controlled rotation stage, so that the transverse position of the feedback beam in the crystal can be varied with

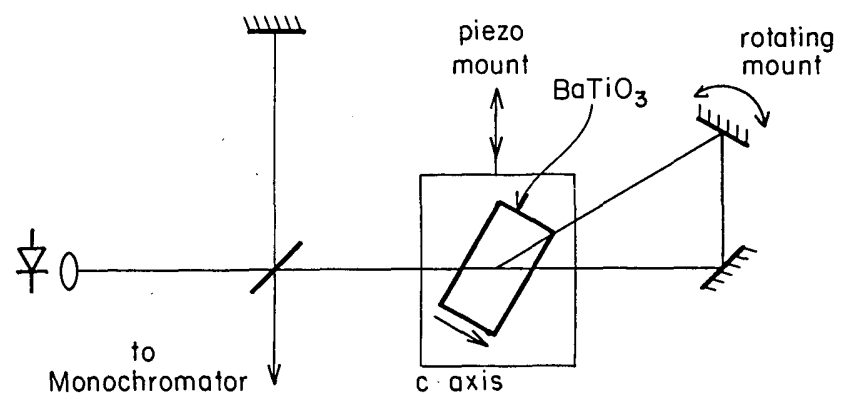

Fig. 1. Schematic of experiment showing frequency scanning in a GaAlAs laser with photorefractive phase-conjugate feedback. 


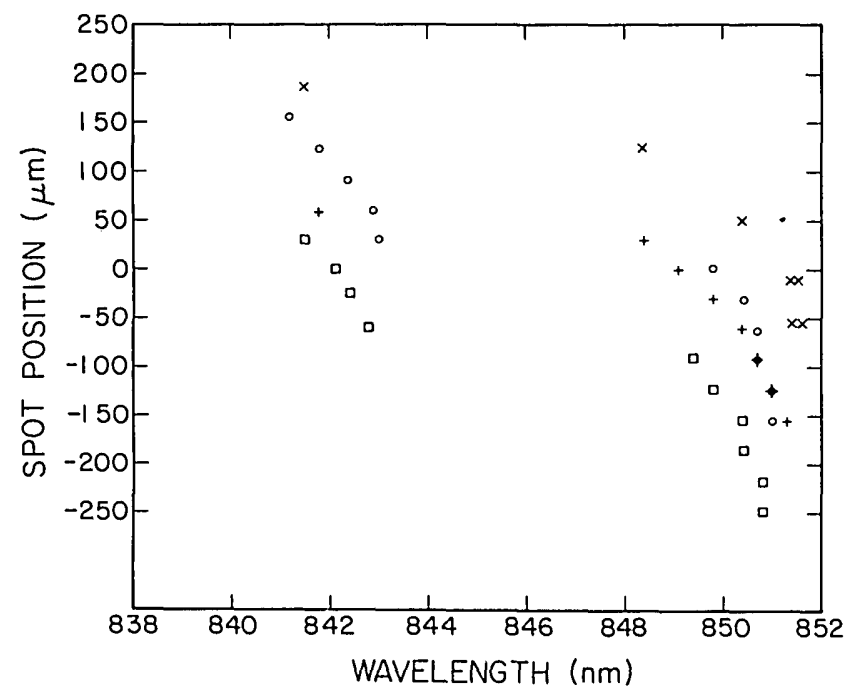

Fig. 2. Center frequency of diode-laser spectrum versus transverse position of the feedback beam in the crystal with respect to the position of the incident beam. Positive displacement corresponds to counterclockwise rotation of the adjustable-feedback mirror. Different data point forms (crosses, circles, etc.) correspond to different experimental runs.

respect to the position of the incident beam. The crystal itself is mounted on a platform that can be piezoelectrically translated to simulate spontaneously moving photorefractive index gratings. To reduce Fresnel reflection losses at its surfaces, the crystal is immersed in an oil-filled cuvette. The laser intensity is measured by a detector monitoring a reflection from one of the cuvette surfaces. The reflectivity of the PPCM in steady state was about $17 \%$, uncorrected for Fresnel reflections.

The frequency-scanning behavior of this system was found to be dependent principally on the alignment of the ring PPCM, adjusted by the rotatable feedback mirror. For small misalignments, the laser spectrum scans once to a center frequency determined by the direction and amount of the misalignment. It then stabilizes, often in one, but sometimes in two or three, longitudinal modes. This can be explained by the Bragg selectivity of the photorefractive index gratings, which is about $1 \mathrm{~nm}$, encompassing about three longitudinal modes of the laser. Figure 2 shows the settling frequency of the laser versus tranverse position of the feedback beam in the crystal with respect to the position of the incident beam. Clockwise rotation of the adjustable mirror leads to a red shift; counterclockwise rotation leads to a blue shift. Note that there seems to be an excluded band in the center of the range. When the ring PPCM is misaligned just beyond the range shown in Fig. 2, repetitive frequency scans take place. Figures 3(a) and 3(b) show the center frequency and laser output intensity during a series of three consecutive red scans and five consecutive blue scans, respectively. As the laser frequency is dragged farther away from its preferred operating point, the output intensity decreases until the laser is detuned to such a degree that, in spite of the Bragg- selected feedback at the distant frequency, it begins to lase again at its preferred operating frequency. The light at this frequency is not Bragg matched to the grating in the crystal and thus erases it, causing the ring PPCM to cease operation. New gratings build up at the preferred frequency and another scan begins. Note that the observed scan rate for the blue scans is $0.2 \mathrm{~nm} / \mathrm{sec}$ or $84 \mathrm{GHz} / \mathrm{sec}$.

We recently operated the cat mirror with the same GaAlAs lasers and saw much slower nonrepetitive frequency scanning toward the red. The coupling constant threshold for the cat mirror is higher than for the ring mirror, and to obtain its operation we thermoelectrically cooled the crystal toward its tetragonal to orthorhombic phase transition at round $9^{\circ} \mathrm{C}$ to take advantage of its higher electro-optic coefficient there. ${ }^{11}$ The reflectivity $R$ is given approximately by $R=7-$ $0.45\left(T-T_{0}\right) \%$, and the settling center wavelength of the laser is $\lambda=850-0.3\left(T-T_{0}\right) \mathrm{nm}$, where $T_{0}$ is the transition temperature. At $10^{\circ} \mathrm{C}$ the scan rate is 0.03 $\mathrm{nm} / \mathrm{sec}$. The spectral narrowing effect was not so clearly evident in the case of the cat mirror as in the ring mirror, perhaps because of the relative smallness of the cat mirror reflectivity.

It has been suggested that the scanning effects in dye lasers are caused by accumulation of Doppler shifts from reflection from spontaneously moving gratings in the photorefractive crystal. ${ }^{3}$ We have sev-

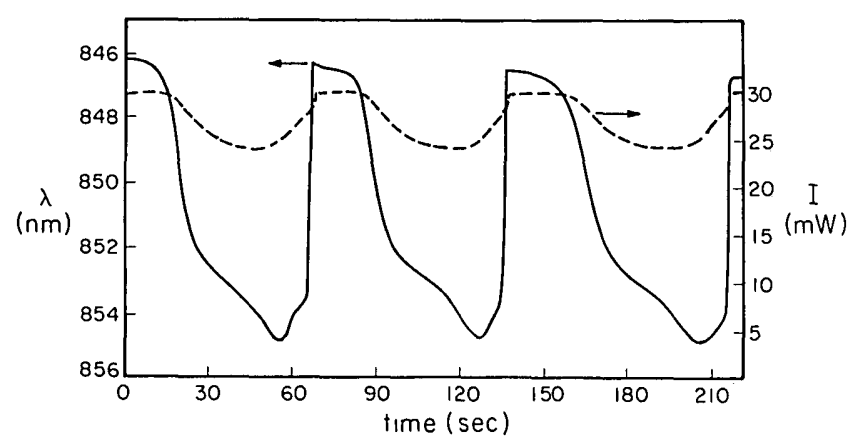

(a)

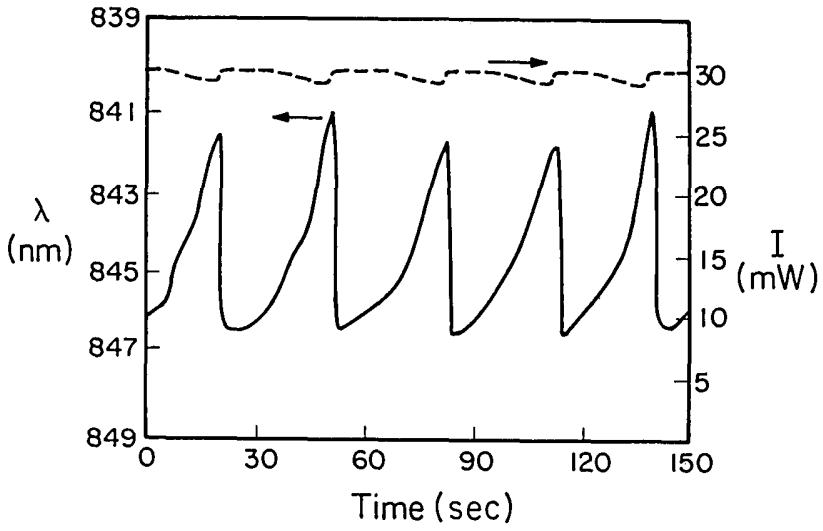

(b)

Fig. 3. Laser output intensity (dashed line) and center frequency (solid line) during several consecutive repetitive (a) red-shifting and (b) blue-shifting scans. 
eral reasons to believe that this is not the case for selfscanning diode lasers. If the light were Doppler shifted each round trip in the external cavity, then the laser would be expected to scan $\Delta f \mathrm{~Hz}$ every $2 L / c$ sec, where $\Delta f$ is the Doppler shift associated with reflection from the moving gratings and $L$ is the external cavity length, taken to be half of the round-trip distance traveled by light from the diode to the crystal, around the PPCM ring, and back to the diode. The scanning rate of the laser would then be $c \Delta f / 2 L \mathrm{~Hz} / \mathrm{sec}$. In our case, $L$ is $24 \mathrm{~cm}$, and the observed scan rate for the blue scans in the ring mirror is $0.2 \mathrm{~nm} / \mathrm{sec}$. To explain this scan rate by cumulative Doppler shifts would require $\Delta f \approx 130 \mathrm{~Hz}$. First, this is well beyond the frequency response $(\approx 1 \mathrm{~Hz})$ of the crystal at the optical intensities that we have used $\left(\approx 5 \mathrm{~W} / \mathrm{cm}^{2}\right)$. The corresponding result for the cat mirror red scans is $0.03 \mathrm{~nm} / \mathrm{sec}$, requiring $\Delta f \approx 20 \mathrm{~Hz}$. Second, if $\Delta f$ were different from zero, inspection of the interferometer output where the direct laser light interferes with light that has been Doppler shifted in the phase-conjugate mirror by an amount $\Delta f$ would reveal fringe movement. The frequency with which these fringes move past a fixed point would be a direct measurement of $\Delta f$. No such movement is observed in our experiment, neither for the ring mirror nor for the cat mirror. The experimental uncertainty of this observation is much less than $1 \mathrm{~Hz}$. Third, the detuning behavior is unaffected by moving the crystal on its piezomount to simulate moving gratings, artificially inducing a nonzero $\Delta f$, which was easily monitored by observing motion of the interference fringes. All these observations tend to rule out a Doppler shift from a moving grating as the origin of the self-scanning in diode lasers. One of the reviewers has suggested that the observed rate of frequency scanning may be caused by very slowly moving gratings if a cleaved coupled cavity type of effect is operative, with the two cavities in this case being the actual semiconductor laser cavity and the external cavity bounded by the phase-conjugate mirror. The fact that piezoelectrically moving the grating does not influence the scanning rate would seem to rule out this possibility. We are currently carrying out more-extensive experiments designed to determine the origin of the scanning behavior. One possibility, suggested by the alignment sensitivity, is that it is caused by a directional dependence in the Bragg frequency selectivity of the photorefractive gratings.

In summary, we have observed frequency scanning and spectral narrowing in semiconductor lasers with photorefractive self-pumped phase-conjugate feedback. The amount and direction of scanning caused by feedback in the case of the ring mirror can be controlled by adjustments in alignment of the phaseconjugate mirror.

The research was supported by the U.S. Army Research Office, Durham, North Carolina.

\section{References}

1. W. B. Whitten and J. M. Ramsey, Opt. Lett. 9, 44 (1984).

2. F. C. Jahoda, P. G. Weber, and J. Feinberg, Opt. Lett. 9, 362 (1984).

3. J. Feinberg and G. D. Bacher, Opt. Lett. 9, 420 (1984).

4. J. M. Ramsey and W. B. Whitten, Opt. Lett. 10, 362 (1985).

5. J. O. White, M. Cronin-Golomb, B. Fischer, and A. Yariv, Appl. Phys. Lett. 40, 450 (1982).

6. J. Feinberg, Opt. Lett. 7, 486 (1982).

7. M. Cronin-Golomb, B. Fischer, J. O. White, and A. Yariv, IEEE J. Quantum Electron. QE-20, 12 (1984).

8. J. M. Ramsey and W. B. Whitten, Anal. Chem. 56, 2979 (1984).

9. M. Cronin-Golomb, K. Y. Lau, and A. Yariv, Appl. Phys. Lett. 47, 567 (1985).

10. K. Y. Lau, N. Bar-Chaim, I. Ury, and A. Yariv, Appl. Phys. Lett. 45, 316 (1984).

11. M. Cronin-Golomb, G. Rakuljic, and A. Yariv, Proc. Soc. Photo-Opt. Instrum. Eng. (to be published). Although the temperature of the tetragonal to orthorhombic phase transition is usually published as $5^{\circ} \mathrm{C}$, we have found that the transition temperature in our crystals is closer to $9^{\circ} \mathrm{C}$. 